\title{
Aceptación de los dispositivos de bioseguridad de material corto-punzante en personal de enfermería de un hospital terciario
}

\author{
Acceptance of safety devices \\ in nursing staff in general hospital \\ Lourdes Jiménez Bajo \\ Cristina Serrano Ramos \\ María Luisa Valle Robles \\ Ignacio Bardón Fernández-Pacheco \\ Silvia O'Connor Pérez \\ Covadonga Caso Pita \\ Servicio de Prevención de Riesgos Laborales \\ de Hospital Clínico San Carlos \\ Correspondencia: \\ Lourdes Jiménez Bajo \\ Hospital Clínico San Carlos \\ Profesor Martín Lagos S/N \\ 28040 Madrid \\ jimenezbajol@hotmail.com
}

Resumen

El objetivo de este estudio es evaluar la aceptación entre el personal de enfermería de los dispositivos de bioseguridad de material corto-punzante tras 20 meses de su implantación en un hospital terciario y su comparación con los resultados obtenidos en el estudio piloto previo a la implantación.

Se realiza estudio descriptivo transversal en 5 servicios del hospital, Nefrología, Medicina Interna, Infecciosas, Unidad de Críticos y Cirugía General antes y tras 20 meses de implantación de los dispositivos. El cuestionario recoge variables como seguridad de usuario, comodidad y sencillez de uso, tiempo de maniobra y seguridad del paciente de cinco dispositivos, agujas intramusculares, agujas subcutáneas, jeringas de gasometría, catéter intravenoso y aguja de diálisis; valoración global de la importancia del uso generalizado de los dispositivos y necesidad de más información/ formación.

El 62\% considera importantísimo el uso generalizado de dispositivos de seguridad.

Tras 20 meses de implantación, la aceptación de los dispositivos entre el personal de enfermería parece mayor, aunque estas diferencias no son estadísticamente significativas. Es necesario aumentar el período de estudio y reforzar las medidas de información-formación entre otras propuestas, ya que contribuyen a su mayor aceptación la frecuencia de utilización y la información/ formación recibida.

(Med Segur Trab 2009; 55 (215): 19-27)

Palabras clave: aceptación, dispositivos de bioseguridad, personal de enfermería, informaciónformación. 


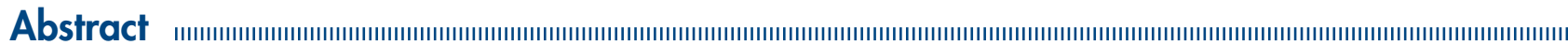

The purpose of this study is asses the acceptance of the safety devices in nursing staff after 20 months of implementation in general hospital and compare the results with pilot study before implementation.

A descriptive study was conducted in 5 hospital services: Nephrology, Internal Medicine, Infectous Diseases Service, Intensive Care and General Surgery.

The questionnaire includes following variables: user safety, comfort and ease of use, time to maneuver and patient safety of the five devices, intramuscular needle, needle subcutaneous, syringe of gas, intravenous catheters and needle of dialysis; overall assessment of the importance of widespread use of devices and the need for more information.

$62 \%$ of respondents consider import the widespread use of devices.

After 20 months of implementation, the acceptance of the devices among nursing staff appeared to be higher, even these differences are not statistically significant. It is necessary to increase the study period and strengthening the information/ training measures as they contribute to their greater acceptance, the frequency of use and the information received.

(Med Segur Trab 2009; 55 (215): 19-27)

Key words: acceptance, safety devices, nursing staff, information/training measures. 


\section{INTRODUCCIÓN}

Las inoculaciones accidentales con material corto-punzante continúan siendo una fuente de exposición a patógenos importantes como virus de la hepatitis B, virus de la hepatitis C y de VIH en profesionales sanitarios.

Para prevenir este tipo de accidentes se han diseñado dispositivos con mecanismos de bioseguridad que intentan reducir el riesgo de exposiciones percutáneas, encuadrados dentro de un plan integral de prevención de este tipo de lesiones.

La selección de los dispositivos de bioseguridad de material corto-punzante y su evaluación posterior irán dirigidas a garantizar su funcionamiento de manera efectiva, que resulten aceptables para el personal sanitario que los utiliza y seguros para el paciente ${ }^{1}$.

La eficacia de los dispositivos en la reducción de inoculaciones accidentales en personal sanitario está ampliamente documentada, al igual que diversos estudios justifican el coste-beneficio de la implantación de este tipo de medida. Sin embargo no existen muchos estudios que analicen específicamente la aceptación de los dispositivos de bioseguridad en el personal sanitario que los utiliza ${ }^{2}$.

En la aceptación de los dispositivos intervienen factores como la percepción del riesgo por parte del personal usuario; la percepción de que existe un riesgo elevado de contraer enfermedades importantes a través de exposiciones percutáneas, contribuirá a una mayor aceptación del dispositivo ${ }^{3}$.

Influye de manera determinante la información- formación recibida sobre el uso de los dispositivos antes y después de la implantación de los mismos en los centros sanitarios ${ }^{4}$. Una mayor formación tendrá efectos positivos en la aceptación de esta nueva medida, aunque contribuyen a una menor aceptación si el requerimiento de formación para utilizar el dispositivo es amplia o existe gran diferencia con la aguja tradicional.

El periodo de tiempo en el que el dispositivo ha sido utilizado, afecta de manera que cuanto mayor sea este periodo, probablemente su manejo resulte más fácil para el personal.

La participación de los usuarios en el proceso de selección del dispositivo fomenta la mayor aceptación ${ }^{1}$, pero también es necesario que los usuarios participen en el proceso de evaluación de esta medida, contribuyendo a la detección de dificultades en las diferentes técnicas en las que se utilizan los dispositivos y así poder mejorar el diseño de los mismos.

La implantación de dispositivos de material corto-punzante en la Comunidad de Madrid se realiza en noviembre de 2006. Previo a la implantación, se realizó un estudio piloto $^{5}$ en algunos Hospitales y Centros de Atención Primaria de la Comunidad. Los servicios incluidos en nuestro hospital fueron Medicina Interna, Infecciosas, Nefrología, Unidad de Críticos y Cirugía General, incluyendo sólo al personal de enfermería.

Con el fin de evaluar la aceptación de los dispositivos de bioseguridad por parte del personal de enfermería en los servicios citados, tras 20 meses de implantación de los dispositivos en nuestro hospital y comparar los resultados con los obtenidos en el estudio piloto realizado antes de la implantación, diseñamos este estudio.

\section{MATERIAL Y MÉTODO}

Se diseña un estudio descriptivo en panel cuya población de estudio es el personal de enfermería de los Servicios de Medicina Interna, Infecciosas, Nefrología, Unidad de Críticos y Cirugía General de un hospital terciario de la Comunidad de Madrid.

Esta selección se realiza atendiendo a criterios de frecuencia y repercusión de la inoculación accidental en estos servicios. 
Se realizan dos estudios descriptivos en dos periodos de tiempo, previo a la implantación de dispositivos en noviembre de 2006 (durante la realización del proyecto piloto) y tras 20 meses de la implantación de los dispositivos. En el primer periodo, correspondiente al estudio pilotos participaron un total de 203 enfermeros y enfermeras en el hospital. Globalmente, en el estudio piloto participaron un total de 364 sujetos encargados de utilizar y evaluar los nuevos materiales.

En el segundo periodo, tras 20 meses de implantación de los dispositivos, la población diana de nuestro estudio fue todo el personal de enfermería de los servicios que participaron en el estudio piloto 255 enfermeros y enfermeras.

Los dispositivos de bioseguridad que se incluyen en el estudio son agujas intramusculares, agujas subcutáneas, jeringas de gasometría, catéter intravenoso y aguja de diálisis, seleccionados por su importancia y frecuencia de uso.

Los datos se recogen mediante encuesta específica entregada al Supervisor de Enfermería de cada servicio, cumplimentada por el personal de forma anónima y voluntaria y remitida al Servicio de Prevención de Riesgos Laborales.

La encuesta recoge:

- variables profesionales: Servicio al que pertenece, antigüedad profesional, antigüedad en el puesto de trabajo, turno de trabajo y situación laboral.

- variables específicas de cada dispositivo: seguridad de usuario, comodidad de uso, sencillez de uso, tiempo empleado en la maniobra y seguridad del paciente. Las opciones de respuesta son mejor, igual, peor o no procede, comparado con dispositivo tradicional.

- variable general de recomendación de uso de los dispositivos con cuatro opciones: importantísimo, muy recomendable, indiferente o desaconsejable.

- variables referentes a la información-formación recibida y a la necesidad de más información-formación, con cuatro respuestas posibles, nada, algo, bastante y mucho.

La necesidad de una encuesta anónima y autoadministrada como estrategia para evaluar los dispositivos de bioseguridad proporciona la ventaja de ser capaz de llegar a todos los turnos, entre otras razones, pero aporta una limitación, que es el índice bajo de participación, por lo que se intentó la participación de todo el personal de enfermería de los servicios incluidos en el proyecto piloto sin realizar muestreo.

Se diseña una base de datos ad-hoc que incluya todas las variables y se realiza análisis descriptivo de las variables profesionales, recomendación del uso generalizado de los dispositivos y variables referentes a las medidas de información-formación en el periodo de estudio tras 20 meses de implantación de los dispositivos.

Se realiza análisis estadístico mediante método chi cuadrado para comparar la distribución de las variables específicas de cada dispositivo en los dos periodos de tiempo mencionados y valorar si existen diferencias significativas. Se ha considerado significativo valores de $\mathrm{p} \leq 0.05$.

\section{RESULTADOS}

La tasa global de participación en el proyecto piloto, como muestran sus resultados, fue del 69\%, de los 364 participantes, 252 fueron respondedores. En el caso de nuestro hospital la tasa de respuesta fue del 65\%, de los 203 participantes fueron respondedores 131 .

La tasa de respuesta en el segundo periodo fue globalmente del 43\%, de los 255 sujetos de estudio, fueron respondedores 110 sujetos. 
Por servicio encontramos una tasa de respuesta del $56 \%$ en el Servicio de Medicina Interna e Infecciosas, 43\% en Cirugía General, 8\% en Unidad de Cuidados Intensivos y $65 \%$ en Nefrología. Los resultados se muestran en la Tabla I.

Tabla I. Tasa de respuesta por Servicios

\begin{tabular}{lccc}
\hline & Respondedores & n & Tasa de respuesta \\
\hline Medicina Interna- Infecciosas & 48 & 85 & $56 \%$ \\
Cirugía General & 31 & 72 & $43 \%$ \\
UCI & 5 & 57 & $8 \%$ \\
Nefrología & 27 & 41 & $65 \%$ \\
Total & 110 & 255 & $43 \%$ \\
\hline
\end{tabular}

Los resultados obtenidos para cada uno de los dispositivos, comparados con el dispositivo tradicional, antes y después de la implantación de los mismos en nuestro hospital, se recogen en las siguientes tablas.

Los resultados obtenidos para las agujas intramusculares de seguridad se calcularon a partir de 175 encuestas recogidas antes y 108 después de la intervención y muestran como se agrupan los porcentajes de mejor opinión sobre el dispositivo de seguridad implantado comparado con el tradicional. Los resultados se muestran en la Tabla II.

Tabla II. Agujas intramusculares de seguridad

\begin{tabular}{|c|c|c|c|c|}
\hline & & mejor & igual & peor \\
\hline \multirow{2}{*}{$\begin{array}{l}\text { Seguridad usuario } \\
\text { X2:0.758 }\end{array}$} & antes & $81 \%$ & $9 \%$ & $10 \%$ \\
\hline & después & $82 \%$ & $10 \%$ & $8 \%$ \\
\hline \multirow{2}{*}{$\begin{array}{l}\text { Comodidad usuario } \\
\text { X2:0.113 }\end{array}$} & antes & $20 \%$ & $31 \%$ & $47 \%$ \\
\hline & después & $30 \%$ & $23 \%$ & $46 \%$ \\
\hline \multirow{2}{*}{$\begin{array}{l}\text { Sencillez uso } \\
\mathrm{X} 2: 0.422\end{array}$} & antes & $26 \%$ & $55 \%$ & $24 \%$ \\
\hline & después & $31 \%$ & $50 \%$ & $18 \%$ \\
\hline \multirow{2}{*}{$\begin{array}{l}\text { Tiempo maniobra } \\
\text { X2:0.105 }\end{array}$} & antes & $15 \%$ & $65 \%$ & $20 \%$ \\
\hline & después & $24 \%$ & $60 \%$ & $16 \%$ \\
\hline \multirow{2}{*}{$\begin{array}{l}\text { Seguridad paciente } \\
\text { X2:0.003 }\end{array}$} & antes & $11 \%$ & $77 \%$ & $11 \%$ \\
\hline & después & $24 \%$ & $70 \%$ & $4 \%$ \\
\hline
\end{tabular}

De la misma forma observamos las opiniones de los trabajadores en cuanto a satisfacción se refiere comparando las agujas subcutáneas de seguridad con las antiguas agujas. Los resultados se muestran en la Tabla III. Las encuestas que se recogieron ascienden a 201 antes y 110 después. 
Tabla III. Agujas subcutáneas de seguridad

\begin{tabular}{lllll}
\hline & & mejor & igual & peor \\
\hline Seguridad usuario & antes & $83 \%$ & $11 \%$ & $6 \%$ \\
X2:0.0347 & después & $93 \%$ & $6 \%$ & $1 \%$ \\
Comodidad usuario & antes & $20 \%$ & $47 \%$ & $33 \%$ \\
X2:0.028 & después & $35 \%$ & $37 \%$ & $28 \%$ \\
Sencillez uso & antes & $20 \%$ & $61 \%$ & $18 \%$ \\
X2:0.014 & después & $34 \%$ & $54 \%$ & $12 \%$ \\
Tiempo maniobra & antes & $15 \%$ & $70 \%$ & $15 \%$ \\
X2:0.067 & después & $26 \%$ & $62 \%$ & $12 \%$ \\
Seguridad paciente & antes & $10 \%$ & $81 \%$ & $9 \%$ \\
X2:6.10E-O5 & después & $27 \%$ & $70 \%$ & $3 \%$ \\
& & & & \\
\hline
\end{tabular}

En el caso de las jeringas de seguridad para realizar gasometría recogemos 98 encuestas antes y 107 después. Los resultados aparecen en la Tabla IV de comparación de porcentajes en las diferentes categorías.

Tabla IV. Jeringas con aguja de seguridad para gasometría

\begin{tabular}{lllll}
\hline & & mejor & igual & peor \\
\hline $\begin{array}{llll}\text { Seguridad usuario } \\
\text { X2:0.342 }\end{array}$ & antes & $83 \%$ & $13 \%$ & $4 \%$ \\
& después & $85 \%$ & $13 \%$ & $2 \%$ \\
Comodidad usuario & antes & $21 \%$ & $50 \%$ & $27 \%$ \\
X2:0.144 & después & $33 \%$ & $40 \%$ & $26 \%$ \\
Sencillez uso & antes & $21 \%$ & $62 \%$ & $16 \%$ \\
X2:0.082 & después & $35 \%$ & $55 \%$ & $10 \%$ \\
Tiempo maniobra & antes & $13 \%$ & $72 \%$ & $14 \%$ \\
X2:0.048 & después & $27 \%$ & $63 \%$ & $10 \%$ \\
Seguridad paciente & antes & $11 \%$ & $81 \%$ & $7 \%$ \\
X2:0.013 & después & $26 \%$ & $72 \%$ & $2 \%$ \\
\hline
\end{tabular}

Las opiniones del personal de enfermería aportadas en cuanto a catéteres intravenosos de seguridad no muestran apenas diferencias antes y después. Los resultados se han obtenido a partir de 196 encuestas antes y 104 después y se muestran en la Tabla V. 
Tabla V. Catéteres intravenosos de seguridad

\begin{tabular}{lllll}
\hline & & mejor & igual & peor \\
\hline $\begin{array}{llll}\text { Seguridad usuario } \\
\text { X2:1E-O5 }\end{array}$ & antes & $83 \%$ & $4 \%$ & $12 \%$ \\
& después & $81 \%$ & $14 \%$ & $3 \%$ \\
Comodidad usuario & antes & $26 \%$ & $14 \%$ & $62 \%$ \\
X2:1E-08 & después & $40 \%$ & $35 \%$ & $25 \%$ \\
Sencillez uso & antes & $24 \%$ & $27 \%$ & $49 \%$ \\
X2:1.3E-O5 & después & $33 \%$ & $45 \%$ & $21 \%$ \\
Tiempo maniobra & antes & $17 \%$ & $40 \%$ & $43 \%$ \\
X2:8.3E-06 & después & $15 \%$ & $54 \%$ & $16 \%$ \\
Seguridad paciente & antes & $12 \%$ & $44 \%$ & $44 \%$ \\
X2:4.8E-08 & después & $27 \%$ & $61 \%$ & $12 \%$ \\
& & & & \\
\hline
\end{tabular}

Los resultados comparando las agujas de diálisis de seguridad con las anteriormente utilizadas obtenidos a partir de 26 y 27 encuestas en los dos momentos citados, agrupan porcentajes de opinión mejores, iguales o peores antes y después de la siguiente manera, se muestran en la Tabla VI.

Tabla VI. Agujas de diálisis de seguridad

\begin{tabular}{lllll}
\hline & & mejor & igual & peor \\
\hline $\begin{array}{llll}\text { Seguridad usuario } \\
\text { X2:0.854 }\end{array}$ & antes & $94 \%$ & $4 \%$ & $2 \%$ \\
& después & $91 \%$ & $8 \%$ & $1 \%$ \\
Comodidad usuario & antes & $12 \%$ & $39 \%$ & $47 \%$ \\
X2:0.037 & después & $48 \%$ & $22 \%$ & $28 \%$ \\
Sencillez uso & antes & $14 \%$ & $53 \%$ & $33 \%$ \\
X2:0.049 & después & $48 \%$ & $40 \%$ & $12 \%$ \\
Tiempo maniobra & antes & $15 \%$ & $70 \%$ & $15 \%$ \\
X2:0.094 & después & $40 \%$ & $55 \%$ & $5 \%$ \\
Seguridad paciente & antes & $3 \%$ & $56 \%$ & $41 \%$ \\
X2:0.001 & después & $37 \%$ & $59 \%$ & $4 \%$ \\
\hline
\end{tabular}

Los resultados obtenidos no son estadísticamente significativos, en ninguna de las comparaciones realizadas, para cada dispositivo de seguridad y en todas las variables recogidas, considerando significativo valores de $\mathrm{p} \leq 0.05$.

Los resultados obtenidos acerca de la recomendación del uso generalizado de los dispositivos muestran que un $62 \%$ de los encuestados lo considera "importantísimo", un $36 \%$ "muy recomendable" y un $2 \%$ " indiferente".

En cuanto a las variables relativas a la información-formación, un $60 \%$ refiere" no" haber recibido formación, un $20 \%$ algo, un $13 \%$ " bastante" información y un $6 \%$ "mucha". 
La necesidad de más información la refieren un $42 \%$ como necesidad de "algo" más de información-formación, un 36\%" bastante", un $11 \%$ "mucha" y un 9\% refieren no necesidad de más información.

\section{CONCLUSIONES}

La implantación de nuevos dispositivos de bioseguridad en el material corto-punzante supone un esfuerzo adicional al trabajador que los utiliza, ya que requiere un periodo de entrenamiento para su adaptación al nuevo material.

En un primer momento, el mecanismo de seguridad de agujas puede resultar más incómodo en su utilización o complicar las maniobras o técnicas para las que está diseñado, hecho que parece disminuir cuando la frecuencia de uso de los dispositivos es mayor $^{3}$.

De la misma forma sucede que el tiempo de maniobra utilizando estos mecanismos de bioseguridad puede verse alargado y será la mayor utilización la que acorte este periodo de tiempo ${ }^{3}$.

Las medidas de información-formación son claves antes y después de la implantación de los dispositivos ${ }^{2,3,6}$. Antes por la preparación a la nueva medida, información sobre los riesgos que conlleva la utilización de material corto-punzante, la prevención de transmisión de patógenos a través de sangre u otros fluidos y cómo los dispositivos de bioseguridad pueden reducir el número de accidentes con riesgo biológico. El conocimiento de los factores implicados en este tipo de accidentes y como la promoción de ciertas prácticas favorecen la efectividad de los dispositivos contribuirán a mejorar la aceptación de éstos ${ }^{1}$.

Se necesita individualizar las medidas de información-formación de cada dispositivo y las técnicas para las que va a ser utilizado, intentando siempre actuar en los colectivos más concienciados con el riesgo biológico y la utilización de material corto-punzante.

Es necesaria la participación activa de los trabajadores en la evaluación de las medidas implantadas a partir de la creación de un equipo multidisciplinar que elabore un plan para reducir los accidentes por pinchazos con material corto-punzante ${ }^{1,3,7}$.

Durante la implantación de los dispositivos debe fomentarse su evaluación para detectar problemas en su utilización en técnicas o pacientes con características concretas ${ }^{2,3}$ El nuevo dispositivo con mecanismo de seguridad debe resultar aceptable al trabajador sanitario que lo utiliza.

En nuestro estudio la inmensa mayoría de los encuestados considera importantísimo, un $62 \%$ o muy recomendable, un $39 \%$ el uso generalizado de este tipo de dispositivos para prevenir inoculaciones accidentales ${ }^{6}$. Este altísimo porcentaje parece mostrar la concienciación y aceptación en personal de enfermería, de cómo los dispositivos con mecanismos de seguridad intervienen en la prevención de accidentes biológicos con material corto-punzante.

Considerando por separado cada dispositivo y comparando los resultados con estudio piloto parece que los porcentajes de calificación "mejor" en cuanto a seguridad de usuario, comodidad de uso, sencillez de uso, tiempo empleado de maniobra y seguridad del paciente de los dispositivos incluidos en nuestro estudio en comparación con dispositivo tradicional son mayores tras 20 meses de implantación que en estudio piloto.

De la misma forma parecen menores los porcentajes "peor" en la valoración que hace el personal de enfermería de los nuevos dispositivos comparando con la aguja tradicional.

La distribución que sigue la valoración por parte del personal de enfermería de la aceptación de cada dispositivo por separado, sugiere un posible cambio respecto a los 
resultados del estudio piloto, la distribución agrupa mejores opiniones en cada una de las características recogidas y reduce las valoraciones negativas sobre la utilización de este tipo de agujas.

Estos resultados no son estadísticamente significativos, por lo que parece necesario incluir un mayor periodo de estudio, ya que influye de manera decisiva en la aceptación la frecuencia de uso de los dispositivos ${ }^{3}$. El periodo de 20 meses de implantación de esta medida puede no ser suficiente en algunos de los dispositivos incluidos en el estudio para encontrar diferencias significativas, aunque hemos seleccionado los que más se utilizan para intentar evitar un corto periodo de estudio ${ }^{3,5}$.

Otro factor a tener en cuenta que podría estar implicado en la aceptación de los dispositivos en el personal encuestado es la necesidad de mayores medidas de informaciónformación, ya que refieren esta necesidad aproximadamente un 51\% en gran medida. Se nos plantea la necesidad de un refuerzo en la formación tanto para el personal que participó en el estudio piloto, en el que el uso de dispositivos plantea dificultades propias del manejo que surgen posteriormente a la implantación de la medida como para el personal de nueva incorporación que no participó en las medidas de informaciónformación previas a la implantación de noviembre de 2006.

Entre las limitaciones de este estudio se encuentra el índice bajo de participación, aún realizando seguimiento para aumentar la tasa de respuesta. No todo el personal de enfermería de los servicios incluidos fue respondedor. Los sujetos a partir de la entrega de la encuesta son seleccionados por ellos mismos, por lo que estos resultados pueden verse influidos por esta situación .Esto podría deberse a diferencias de turno o antigüedad laboral o Servicio al que pertenecen, entre otras razones, por lo que es necesario un nuevo seguimiento para encontrar, si existen, estas diferencias.

\section{BIBLIOGRAFÍA}

1. Prevención de lesiones por pinchazos en entornos clínicos. Nacional Institute for Occupational Safety and Health (NIOSH, CDC).Publication No 2000-108.Noviembre 1999.

2. Ihrigh M, Cookson ST, Campbell K, Hartstein AI, Jarvis WR. Evaluation of the acceptability of a needles vascular-acces system by nurses. Am Infect Control 1997; 25(5): 434-8.

3. Simpkins SM, Haiduven DJ, Stevens DA. Safety product evaluation: six years of experience. Am Infect Control. 1995 Oct; 23 (5): 317-22

4. Yang YH, Lion SM The effectiveness of a training program on reducing needlestick injuries/ sharp object injuries among soongraduate vocational nursing school students in Southern Taiwan. J Occup Health 2007; 49(5): 424-9.

5. Actuaciones de Bioseguridad para prevenir inoculaciones accidentales en el personal sanitario de la Comunidad de Madrid. Dirección General de Salud Pública y Alimentación de la Comunidad de Madrid.

6. Casey AL, Elliott TS. The usability and acceptability of a needleless connector system. Br J Nurs $2007 \mathrm{Mar}$ 8-21; 16 (5): 267-71.

7. Zanni GR, Wick JY. Preventing needlestick injuries. Consult Pharm 2007, 22(5).

8. Lawrence LW, Delclos GL, Felknor SA, Jonson PC, Frankowski RF, Cooper SP, Davidson A. The effectivenesss of a needleless intravenous conecction system: an assesment by injury rate and user satisfaction. Infect Control Hosp Epidemiol 1997 Mar; 18(3): 175-82.

!|น, 